\title{
Long Noncoding RNA LINC00I73 Contributes to the Growth, Invasiveness and Chemo-Resistance of Colorectal Cancer Through Regulating miR-765/PLP2 Axis
}

This article was published in the following Dove Press journal:

Cancer Management and Research

\begin{abstract}
Yanhui $\mathrm{Yu}^{\mathrm{l}, *}$
Xiuyun $\mathrm{Lu}^{2} *$

Chuandong Yang ${ }^{3}$

Fengxiang Yin ${ }^{4}$

'Physical Examination Center, The 2nd Affiliated Hospital of Harbin Medical University, Harbin I5008I, People's Republic of China; ${ }^{2}$ Department of Traditional Chinese Medicine, The 2nd Affiliated Hospital of Harbin Medical University, Harbin 15008I, People's Republic of China; ${ }^{3}$ Department of Orthopedics, The 4th Affiliated Hospital of Harbin Medical University, Harbin I5000I, People's Republic of China; ${ }^{4}$ Department of Integrated Traditional Chinese Medicine and Western Medicine, Harbin Chest Hospital, Harbin I50056, People's Republic of China

*These authors contributed equally to this work
\end{abstract}

Background: Long noncoding RNA has been involved in tumorigenesis of colorectal cancer (CRC). This study aimed to illustrate the functions and mechanisms of LINC00173 in CRC progression.

Methods: The expression of LINC00173 in CRC tissues and cell lines were analyzed via qRT-PCR. Kaplan-Meier curve was used to determine survival rate. Luciferase reporter assay was conducted to evaluate the interactions among LINC00173, miR-765 and PLP2 (proteolipid protein 2). CCK8 assay, EdU assay, transwell assay and xenograft assay were performed to examine the effect of LINC00173/miR-765/PLP2 axis on proliferation, migration and invasion. The Ki67 expression level in tumors tissues was detected through immunofluorescence assay.

Results: LINC00173 expression was markedly upregulated in CRC tissues and cells. High expression level of LINC00173 in CRC patients was correlated with poor prognosis. LINC00173 knockdown inhibited proliferation, migration, invasion and chemo-resistance of CRC cells in vitro. LINC00173 downregulation delayed CRC growth in vivo. LINC00173 interacted with miR-765 to promote PLP2 expression.

Conclusion: Our results demonstrated that LINC00173 plays an important oncogenic role in CRC via modulating miR-765/PLP2 axis. And LINC00173 may be a potential prognostic biomarker and therapeutic target for CRC.

Keywords: LINC00173, miR-765, PLP2, colorectal cancer

\section{Introduction}

Colorectal cancer (CRC) is one of the most common cancers and the second cause of tumor-associated deaths worldwide. ${ }^{1}$ Because of lacking effective diagnostic biomarkers and therapeutic targets, patients with CRC progress rapidly. ${ }^{2}$ Additionally, due to high rate of metastasis, the mortality of CRC is very high. CRC patients with advanced stage display a rather low five-year survival rate. ${ }^{3}$ To date, the major therapeutic strategy is surgery combined with chemotherapy or radiotherapy. However, resistance to chemotherapy or radiotherapy is a major problem for most CRC patients. ${ }^{4}$ In order to improve the prognosis, it is urgently required to understand the molecular mechanism of CRC progression.

Long non-coding RNAs (lncRNA) are a family of noncoding RNAs with over 200 nucleotides in length which lack protein-coding potential. ${ }^{5}$ LncRNAs have
Correspondence: Yanhui Yu

Physical Examination Center, The 2nd Affiliated Hospital of Harbin Medical University, Harbin I5008I, People's Republic of China

Email yanhuiyuaaa@sina.com 
been demonstrated to exert various functions in cancer, including regulating proliferation, invasion, survival and differentiation. ${ }^{6}$ Dysregulation of lncRNA has been observed in numerous human cancers. ${ }^{7}$ For example, IncRNA LUCAT1 is upregulated in ovarian cancer and promotes tumor growth and invasion via regulating miR612/HOXA13 axis. $^{8}$ LncRNA SNHG16 promotes proliferation, migration and invasion of bladder cancer cells through targeting miR-98/STAT3 signaling. ${ }^{9}$ In addition, IncRNA HULC is overexpressed in liver cancer and contributes to tumor growth and metastasis by elevating HMGA2 level through targeting miR-186. ${ }^{10}$ Thus, it is important to explore the correlation between IncRNA and CRC development.

LINC00173 (Chromosome 12: 116533422.116536513) in chro has been shown to regulate tumorigenesis of lung cancer and breast cancer. ${ }^{11,12}$ However, the exact role of LINC00173 in CRC is unclear. In our study, we showed that LINC00173 is overexpressed in CRC tissues and predicted poor prognosis. LINC00173 knockdown suppressed the proliferation, migration and invasion and increased the sensitivity of CRC cells to 5-fluorouracil (5-FU). Moreover, LINC00173 knockdown inhibited CRC growth in vivo. Mechanistically, we demonstrated that LINC00173 targeted miR-765/PLP2 axis. In conclusion, our findings reveal that LINC00173 exerts oncogenic roles and may be a potential therapeutic target for CRC treatment.

\section{Materials and Methods Human Samples}

Human tissues and adjacent normal tissues (at least $5 \mathrm{~cm}$ away from the cancer tissues) were collected from The 2nd Affiliated Hospital of Harbin Medical University. At least 3 experienced pathologists confirmed all tissue specimens. All tissues were not treated with radiotherapy or chemotherapy before surgery. This study was approved by the Ethics Committee of The 2nd Affiliated Hospital of Harbin Medical University. Written informed consents were provided by patients. All experiments were performed in accordance with the Declaration of Helsinki.

\section{Cell Culture}

Human colon immortalized cell line FHC and CRC cell lines were purchased from The Cell Bank of Type Culture Collection of the Chinese Academy of Sciences. All cells were cultured using DMEM medium (Gibco, USA) containing 10\% FBS (Gibco, USA).

\section{qRT-PCR}

Total RNA was isolated using TRIzol Reagent (Invitrogen; Thermo Fisher Scientific, Inc.) according to the manufacturer's protocol and used for cDNA synthesis through PrimeScript RT Reagent kit (Takara Bio, Inc.). qPCR was performed by using a SYBR Green PCR kit (Takara Bio, Inc.). U6 and GAPDH were utilized as internal controls. Relative expression was calculated according to the $2-{ }^{\Delta \Delta \mathrm{Ct}}$ method. Primer sequences were: LINC00173 (Forward, 5'GCCACCTTGCTCCGCTGTTC-3' and reverse, 5'-CCG AGGCTTGGAGAGGAGG-3'), PLP2 (Forward, 5'-ATTCA TCAACTGGCCCTGGA-3' and reverse, 5'-AACGGGGAA GGTGACATAGG-3') and GAPDH (Forward, 5'-ATGTT GCAACCGGGAAGGAA-3', reverse 5'-AGGAAAAGCA TCACCCGGAG-3').

\section{Cell Transfection}

Short hairpin RNA (shRNA) targeting LINC00173 or PLP2 was utilized to knock down LINC00173 or PLP2. MiR-765 mimics, miR-765 inhibitors and negative controls were synthesized by GenePharma. Cell transfection was performed by using Lipofectamine ${ }^{\circledR} 2000$.

\section{CCK8 Assay}

CCK8 assay was used to measure cell proliferation. Cells were seeded into the 96-well plates and cultured for $72 \mathrm{~h}$. Then, $10 \mu \mathrm{L}$ of CCK8 solution (Dojindo, Japan) was added into each well and incubated for $2 \mathrm{~h}$. Optical density (OD) values at $450 \mathrm{~nm}$ were determined by a microplate reader (Thermo Scientific, USA).

\section{Wound-healing Assay}

Cells were plated into 6-well plates. When the each well was covered with a single layer of cells, a straight line was evenly created using a $200 \mu \mathrm{L}$ pipette tip. After washed with PBS, the cells were grown at $37^{\circ} \mathrm{C}$ for $24 \mathrm{~h}$. The wound area was observed under a microscope at 0 and $24 \mathrm{~h}$.

\section{Cell Migration and Invasion}

Transwell assay was performed to test migration and invasion. Cells were seeded into the upper chamber filled with serum-free medium. The lower chamber was filled with DMEM medium supplemented with $10 \%$ FBS. After cultured for $24 \mathrm{~h}$, migrated or invaded cells in the lower chamber were fixed with $4 \%$ of paraformaldehyde and stained with $0.1 \%$ crystal violet solution for $30 \mathrm{~min}$. Then, cell photograph was captured using a light microscope. 


\section{Dual-luciferase Reporter Assay}

Bioinformatics was used to predict the interaction between LINC00173 and miR-765 or between miR-765 and PLP2 through miRDB (http://mirdb.org) and TargetScan7 (http:// www.targetscan.org/vert 71/) tools. The wild-type (wt) or mutant (mut: 5'-CUCCUCC-3' mutated to 5'-GAGGAGG-3') sequence of LINC00173 or PLP2 was inserted into pMIRReport vector (Promega). For luciferase reporter assay HCT116 cells were co-transfected with luciferase vector and miR-765 mimics using Lipofectamine 2000. After $48 \mathrm{~h}$, the luciferase activity was measured through the Dual Luciferase kit (Promega) and Renilla luciferase activity was used as control.

\section{Tumor Xenograft Assay}

Six-week female BALB/c nude mice were from Beijing Huafu Kang Biological Technology Co., Ltd. $5 \times 10^{6}$ HCT116 cells (sh-LINC00173 or sh-NC) were subcutaneously injected into the right flank of nude mice ( $\mathrm{n}=5$ for each group). The tumor volumes were measured every 1 week according to the formula: tumor volume $\left(\mathrm{cm}^{3}\right)=$ $\left(\right.$ Leng $\times$ Width $\left.^{2}\right) / 2$. After 5 weeks, tumor tissues (five tumor tissues per group from five mice) were harvested and weighed. The animal experiments were approved by The Ethics Committee of The 2nd Affiliated Hospital of Harbin Medical University. All animal operations were performed in accordance with the Animal Policy and Welfare Committee of The 2nd Affiliated Hospital of Harbin Medical University.

\section{Statistical Analysis}

Statistical analysis was conducted using SPSS version 20.0 (IBM, Corp.) results are presented as the mean \pm standard deviation. Differences between groups were analyzed using a Student's $t$-test or a one-way ANOVA. The Kaplan-Meier method was used to draw survival curve and the Log-rank test was used to determine statistical significance. $\mathrm{P}<0.05$ was considered as statistically significant.

\section{Results}

\section{LINC00I73 is Upregulated in CRC}

To analyze LINC00173 expression, we collected 57 pairs of CRC tissues and corresponding normal tissues. Through qRT-PCR, we found that LINC00173 expression was significantly upregulated in CRC tissues (Figure 1A). Besides, LINC00173 expression was also elevated in CRC cell lines compared to FHC cells (Figure 1B). Then, the CRC tissues were divided into two subgroups (high or low expression by using the median value of LINC00173 as cut-off) according to LINC00173 expression values. The overall survival rate was analyzed through Kaplan-Meier curve. The result showed that LINC00173 low expression was associated with high survival rate (Figure 1C). Moreover, we found that LINC00173 high expression was associated with advanced TNM stage and lymph node metastasis (Table 1).

\section{LINC00I73 Targets miR-765/PLP2 Signaling}

We then determined the subcellular location of LINC00173 by qRT-PCR. We found that LINC00173 was mainly expressed in the cytoplasm of HCT116 cells (Figure 2A), suggesting it may be a competing endogenous RNA. Through bioinformatics analysis, we identified miR-765 as the most potential target miRNA (Figure 2B). Moreover, we also predicted the target of miR-765 and identified PLP2 (Figure 2B). To validate it, we performed luciferase reporter assay using HCT116 cells. As shown, miR-765 mimics markedly inhibited the activity of LINC00173-wt or PLP2wt vectors (Figure $2 \mathrm{C}$ and $\mathrm{D}$ ), demonstrating their interactions. qRT-PCR showed that LINC00173 knockdown led to increased expression of miR-765 (Figure 2E). And miR-765 mimics also suppressed the expression of PLP2 (Figure 2F).
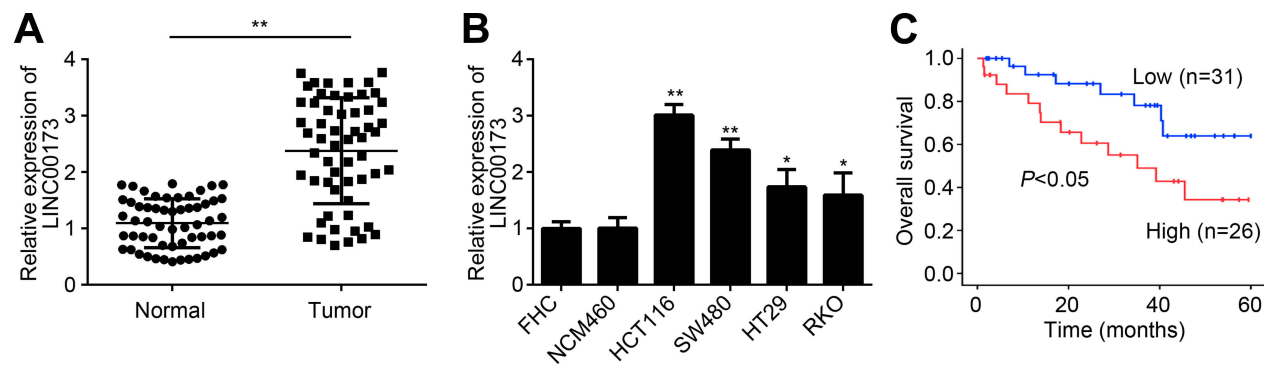

Figure I LINC00I73 is upregulated in CRC. (A) Relative expression of LINC00I73 in 57 pairs of CRC tissues and adjacent normal tissues was analyzed by qRT-PCR. (B) Expression levels of LINC00I73 in CRC cell lines were determined. (C) Survival curve was plotted by using Kaplan-Meier method and log-rank test according to LINC00I73 expression value. $* P<0.05$ and $* * P<0.01$. 
Table I Association Between LINC00173 Expression and Clinical Characteristics in CRC Patients $(n=57)$

\begin{tabular}{|l|l|l|l|}
\hline Characteristics & Low (n=3 I) & High (n=26) & P-value \\
\hline $\begin{array}{l}\text { Gender } \\
\text { Male } \\
\text { Female }\end{array}$ & 17 & 12 & 0.599 \\
\hline $\begin{array}{l}\text { Age (years) } \\
<60\end{array}$ & 14 & 14 & \\
$\geq 60$ & 13 & 13 & 0.600 \\
\hline $\begin{array}{l}\text { Lymph node metastasis } \\
\text { Yes }\end{array}$ & 18 & 13 & \\
No & 21 & 19 & 0.003 \\
\hline $\begin{array}{l}\text { TNM stage } \\
\text { I/II }\end{array}$ & 15 & 7 & \\
III/IV & 16 & 21 & 0.015 \\
\hline
\end{tabular}

Moreover, we found that PLP2 expression was decreased after LINC00173 silencing and rescued after transfection of miR-765 inhibitors (Figure 2G), indicating that LINC00173 promotes PLP2 expression via sponging miR-765.

\section{LINC00173/miR-765/PLP2 Axis Regulates} the Proliferation, Migration, Invasion and Chemo-Resistance of CRC Cells

To further explore the potential roles of LINC00173/miR-765/ PLP2 axis in CRC, we performed a series of experiments. We first measured the expression of PLP2 to confirm the success of transfection (Figure 3A). CCK8 assay showed that LINC00173 knockdown inhibited the proliferation of HCT116 and SW480 cells while miR-765 inhibitors reversed it (Figure 3B). Moreover, PLP2 knockdown further inhibited the proliferation of CRC cells transfected with sh-LINC00173 plus miR-765 inhibitors (Figure 3B), which was further validated by EdU assay (Figure 3C). Then, wound-healing assay was performed to test migration. As shown, in sh-LINC00173 or shLINC00173+miR-inhibitor+sh-PLP2 group the scratch wound closure capacity was obviously suppressed (Figure 3D). Similarly, we measured the effects of LINC00173/miR765/PLP2 axis on metastasis by transwell assay. We found that LINC00173 knockdown inhibited migration and invasion while miR-765 inhibitors reversed it (Figure 3E and F). PLP2 depletion also further reversed it in CRC cells transfected with sh-LINC00173 plus miR-765 inhibitors (Figure 3E and F). To further investigate the effect of LINC00173/miR-765/PLP2 axis on chemo-resistance, we treated HCT116 and SW480 cells with 5-FU after transfection of indicated vectors. Interestingly, we also observed a similar trend as proliferation, migration and invasion (Figure 3G). Therefore, LINC00173 promotes the proliferation, migration, invasion and chemoresistance of CRC cells through targeting miR-765/PLP2 axis.

\section{LINC00 I73/miR-765/PLP2 Axis Affects CRC Growth in vivo}

To further demonstrate their roles in vivo, we performed xenograft assay. We found that LINC00173 knockdown
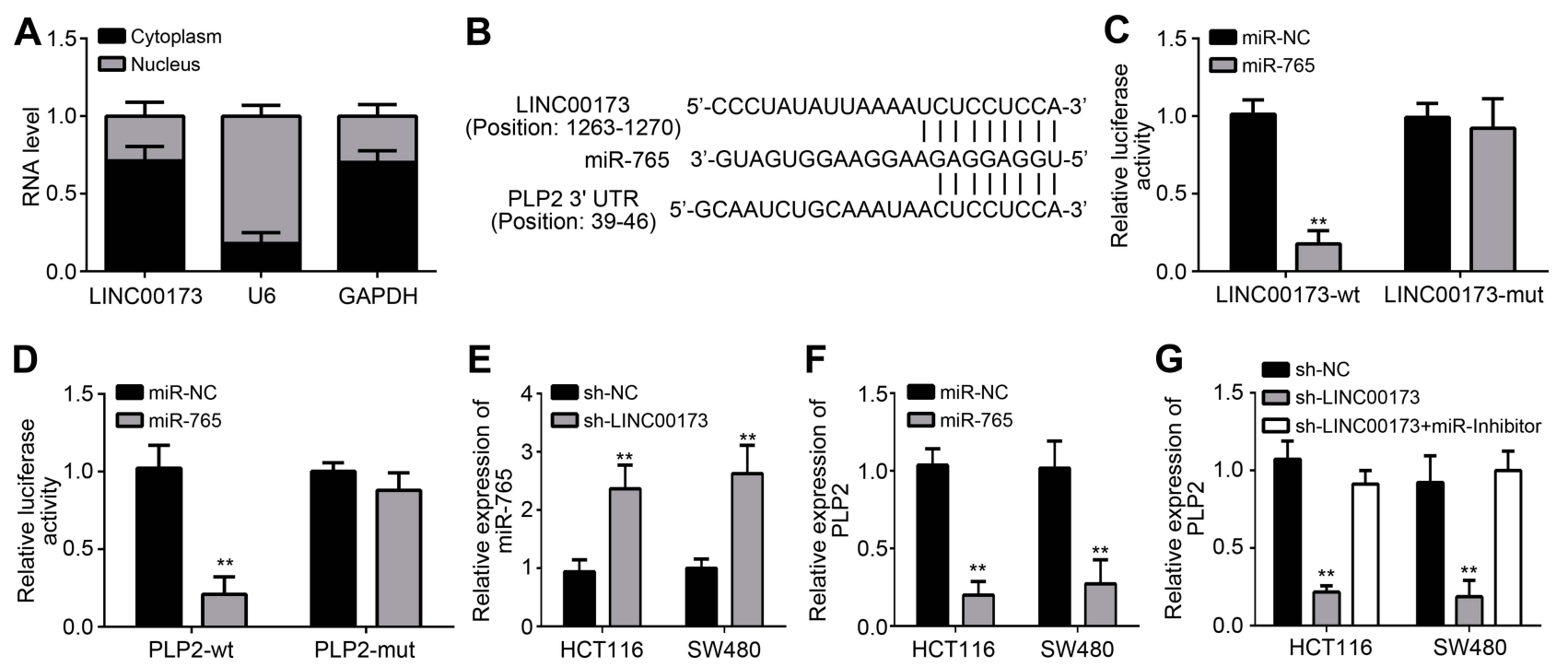

Figure 2 LINC00173 targets miR-765/PLP2 signaling. (A) The subcellular distribution of LINC00I73 in HCTII6 cells was explored. (B) Predicted binding sites among LINC00173, miR-765 and PLP2 were presented. miRDB and TargetScan7 were used for prediction. (C and D) Luciferase reporter assays were performed to validate the interaction between LINC00I73 and miR-765 or between miR-765 and PLP2 in HCTII6 cells. (E) LINC00I73 knockdown promoted miR-765 expression. (F) qRT-PCR showed that miR-765 mimics inhibited PLP2 expression. (G) Relative expression of PLP2 was determined by $\mathrm{qRT}-\mathrm{PCR}$. **P<0.01. 

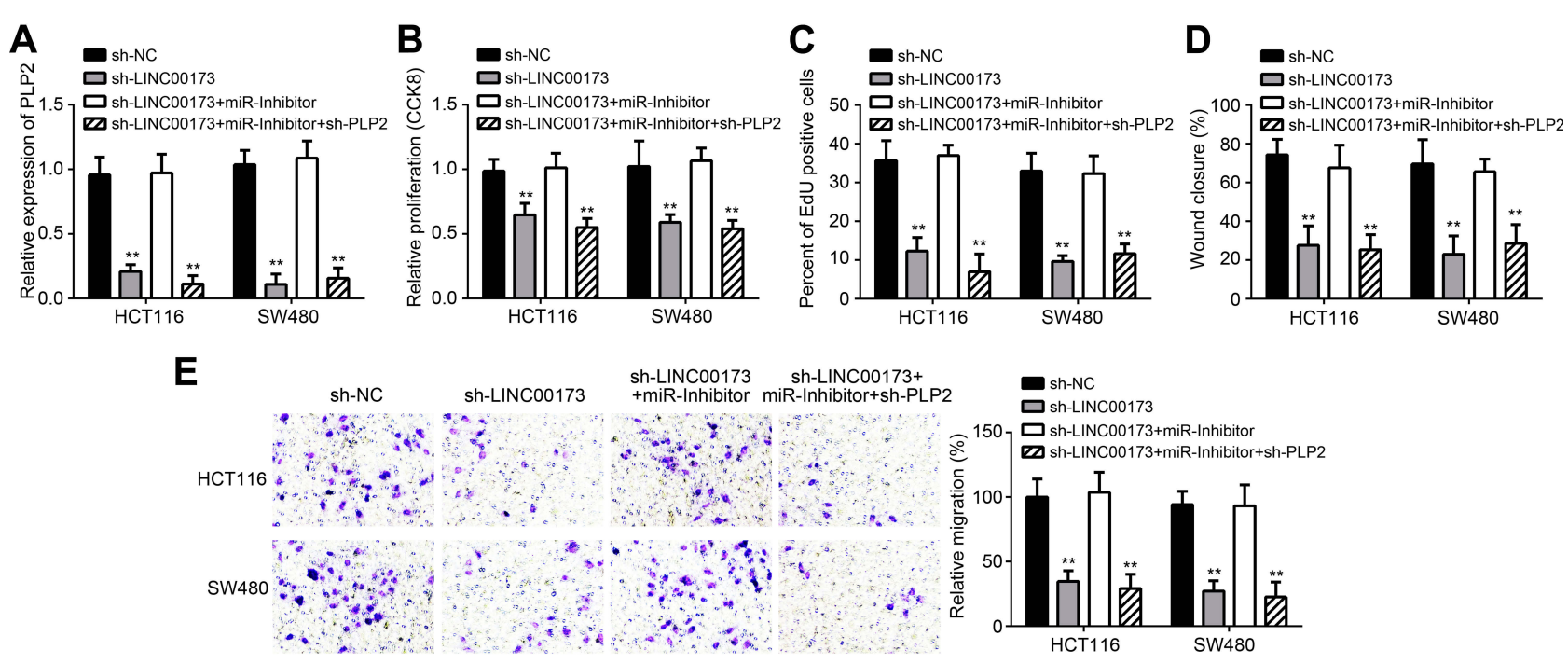

$\mathbf{F}$

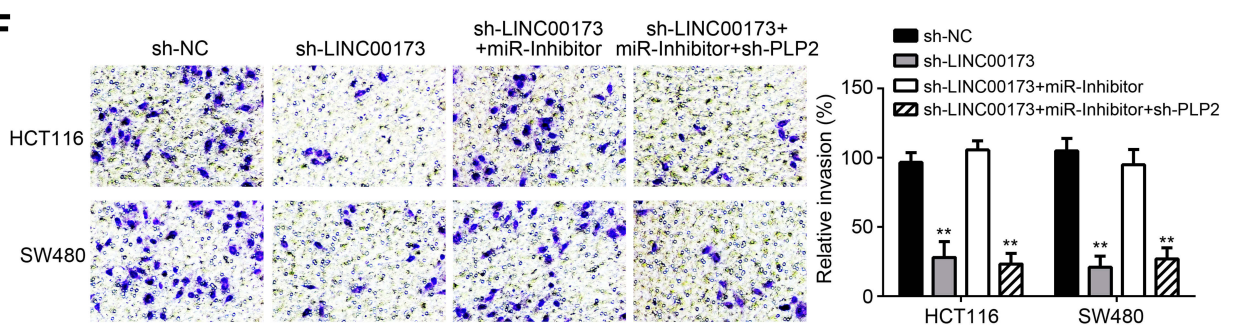

G
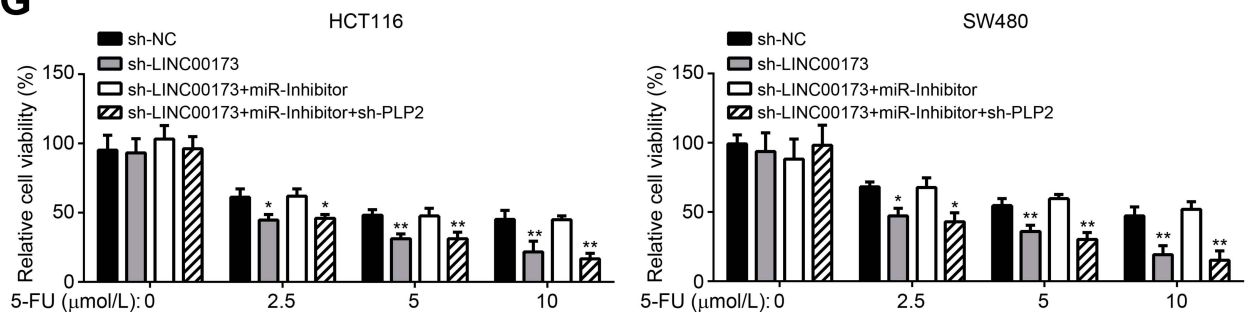

Figure 3 LINC00 I73/miR-765/PLP2 axis regulates the proliferation, migration, invasion and chemo-resistance of CRC cells. (A) Relative expression of PLP2 was determined by qRT-PCR after transfection of indicated plasmids. (B and $\mathbf{C}$ ) CCK8 and EdU assays were performed to detect the proliferation. (D) Cell migration was measured by wound-healing assay. (E and $\mathbf{F}$ ) Transwell assay was performed to analyze cell migration and invasion. (G) The sensitivity to 5 -FU of $C R C$ cells was measured. $* P<0.05$ and $* * P<0.01$.

significantly decreased the tumor volumes and weight (Figure 4A and B). Besides, the Ki67 expression was lower in sh-LINC00173 group than that in control group (Figure 4C). Moreover, we found that the levels of LINC00173 and PLP2 were still downregulated in sh-LINC00173 group while miR765 expression was increased (Figure 4D). Consistently, the protein level of PLC2 was also decreased in sh-LINC00173 group (Figure 4E). Thus, LINC00173 promotes CRC growth in vivo through miR-765/PLP2 axis.

\section{Discussion}

$\mathrm{CRC}$ is a common and malignant cancer in gastrointestinal tract. CRC leads to a large number of cancer-related deaths worldwide. ${ }^{13}$ It is very urgent and important to understand the molecular mechanism of CRC development. Herein, we found that LINC00173 expression was upregulated in CRC tissues and cells. And LINC00173 is a potential prognostic biomarker for CRC patients. We showed that LINC00173 knockdown suppressed the proliferation, migration and invasion of CRC cells in vitro and in vivo. Moreover, we also demonstrated that LINC00173 knockdown sensitized CRC cells to 5-FU treatment. Thus, LINC00173 may be a potential target for CRC therapy.

In recent years, increasing researches have demonstrated that lncRNAs participate in the regulation of tumor development and progression, including CRC. ${ }^{14}$ For example, IncRNA LINC00483 contributes to growth and invasion of CRC through regulating FMNL2 expression. ${ }^{15}$ 

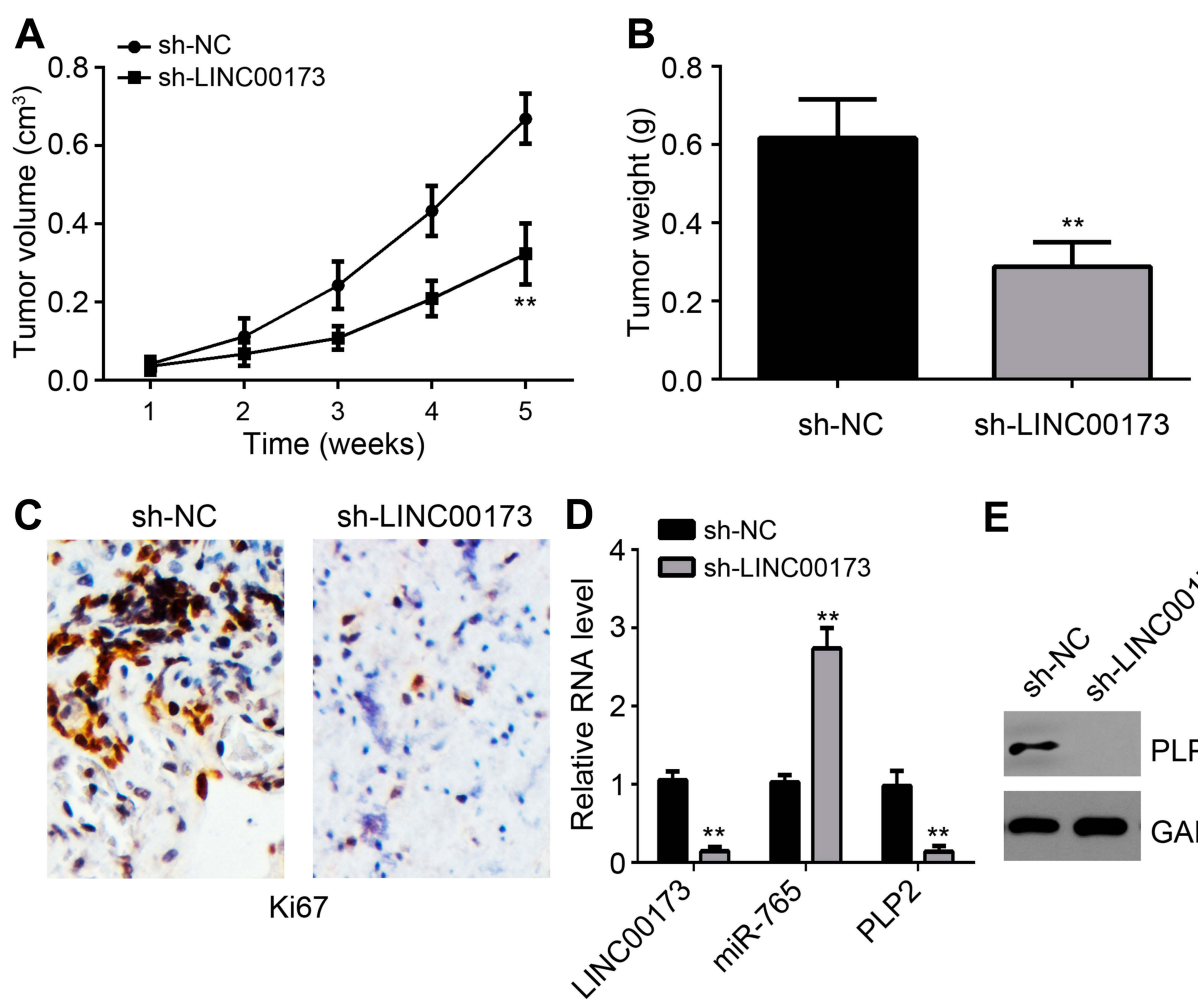

E

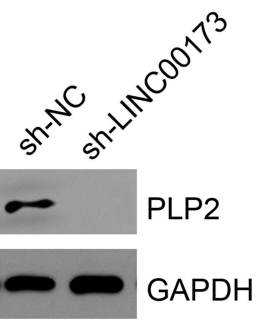

Figure 4 LINC00173/miR-765/PLP2 axis affects CRC growth in vivo. (A) Tumor volumes were determined every I week. (B) Tumor weight was measured after 5 weeks post-injection. (C) Ki67 expression in tumor tissues was examined by IHC assay. (D) Relative expression levels of LINC00I73, miR-765 and PLP2 in tumor tissues were analyzed by qRT-PCR. (E) Protein level of PLP2 was measured by Western blot. **P<0.01.

LncRNA kcna3 is a negative regulator of CRC progression via inhibiting YAP11 expression. ${ }^{16}$ Previously, LINC00173 was reported to promote the proliferation, migration and invasion of lung cancer cells. ${ }^{11}$ Recently, a study indicates that LINC00173contributes to breast cancer progression. ${ }^{12}$ However, its roles in CRC are unclear. In our work, LINC00173 silencing repressed growth and metastasis of CRC cells in vitro and in vivo. Moreover, we also revealed that LINC00173 affected the resistance of CRC cells to 5-FU. Our study demonstrated that LINC00173 is a crucial oncogene in CRC.

Subsequently, we identified that miR-765 is a potential target of LINC00173 through bioinformatics, which was confirmed by luciferase reporter assay. MiR-765 may act as anti-tumor roles or oncogenic functions in different cancer cells. ${ }^{17,18}$ For instance, miR-765 inhibits development of clear cell renal cell cancer via suppressing PLP2 expression. ${ }^{17}$ MiR-765 accelerates growth of liver cancer cells via inhibiting INPP4B expression. ${ }^{18}$ Its function in $\mathrm{CRC}$ remains to be defined. We performed a lot of assays, such as CCK8 assay, EdU assay and Transwell assay. We found that miR-765 inhibitors reversed the effects of
LINC00173 knockdown. Our findings support that miR765 is a suppressor in CRC.

Afterwards, PLP2 was predicted to be targeted by miR765. We also used luciferase reporter assay to confirm the interaction between miR-765 and PLP2. Our finding is consistent with a previous report in clear cell renal cell cancer. ${ }^{17}$ PLP2 has been reported to be involved in several cancers as oncogene. ${ }^{19}$ For example, PLP2 overexpression promotes the development of cutaneous malignant melanoma. ${ }^{19}$ PLP2 upregulation promotes proliferation, migration and invasion of glioma. ${ }^{20}$ Besides, LP2 is also involved in promoting breast cancer cell stemness. ${ }^{21}$ How it functions in CRC is still elusive. In our study, we showed that PLP2 knockdown suppressed the proliferation, migration and invasion of CRC cells. Our results demonstrated that PLP2 promotes CRC development.

In summary, the current work identified that LINC00173 silencing attenuated growth, metastasis and chemo-resistance of CRC cells through sponging miR-765 from PLP2 mRNA. Our study suggests that LINC00173/miR-765/PLP2 axis may be a potential target for CRC therapy. Nevertheless, there are some limitations in the current study. For instance, more CRC 
samples are required to determine association between LINC00173 expression and clinical features. Whether LINC00173 could be a diagnostic biomarker is not defined.

\section{Disclosure}

The authors report no conflicts of interest in this work.

\section{References}

1. Jemal A, Siegel R, Ward E, et al. Cancer statistics, 2008. CA Cancer J Clin. 2008;58(2):71-96. doi:10.3322/CA.2007.0010

2. Siegel RL, Miller KD, Fedewa SA, et al. Colorectal cancer statistics, 2017. CA Cancer J Clin. 2017;67(3):177-193. doi:10.3322/caac.21395

3. Sun X, Suo J, Yan J. Immunotherapy in human colorectal cancer: challenges and prospective. World J Gastroenterol. 2016;22 (28):6362-6372. doi:10.3748/wjg.v22.i28.6362

4. Guo Z, Liu Z, Yue H, Wang J. Beta-elemene increases chemosensitivity to 5-fluorouracil through down-regulating microRNA-191 expression in colorectal carcinoma cells. J Cell Biochem. 2018;119 (8):7032-7039. doi:10.1002/jcb.26914

5. Rao A, Rajkumar T, Mani S. Perspectives of long non-coding RNAs in cancer. Mol Biol Rep. 2017;44(2):203-218. doi:10.1007/s11033017-4103-6

6. Tang Y, He Y, Zhang P, et al. LncRNAs regulate the cytoskeleton and related Rho/ROCK signaling in cancer metastasis. Mol Cancer. 2018;17(1):77. doi:10.1186/s12943-018-0825-x

7. Salehi S, Taheri MN, Azarpira N, Zare A, Behzad-Behbahani A. State of the art technologies to explore long non-coding RNAs in cancer. $J$ Cell Mol Med. 2017;21(12):3120-3140. doi:10.1111/jcmm.13238

8. Yu $\mathrm{H}, \mathrm{Xu}$ Y, Zhang D, Liu G. Long noncoding RNA LUCAT1 promotes malignancy of ovarian cancer through regulation of miR-612/HOXA13 pathway. Biochem Biophys Res Commun. 2018;503(3):2095-2100. doi:10.1016/j.bbrc.2018.07.165

9. Feng F, Chen A, Huang J, Xia Q, Chen Y, Jin X. Long noncoding RNA SNHG16 contributes to the development of bladder cancer via regulating miR-98/STAT3/Wnt/beta-catenin pathway axis. $J$ Cell Biochem. 2018;119(11):9408-9418. doi:10.1002/jcb.27257

10. Wang Y, Chen F, Zhao M, et al. The long noncoding RNA HULC promotes liver cancer by increasing the expression of the HMGA2 oncogene via sequestration of the microRNA-186. J Biol Chem. 2017;292(37):15395-15407. doi:10.1074/jbc.M117.783738
11. Zeng F, Wang Q, Wang S, et al. Linc00173 promotes chemoresistance and progression of small cell lung cancer by sponging miR-218 to regulate Etk expression. Oncogene. 2020;39(2):293-307. doi:10. 1038/s41388-019-0984-2

12. Fan H, Yuan J, Li X, et al. LncRNA LINC00173 enhances triple-negative breast cancer progression by suppressing miR-4903p expression. Biomed Pharmacother. 2020;125:109987. doi:10. 1016/j.biopha.2020.109987

13. Arnold M, Sierra MS, Laversanne M, Soerjomataram I, Jemal A, Bray F. Global patterns and trends in colorectal cancer incidence and mortality. Gut. 2017;66(4):683-691. doi:10.1136/gutjnl-2015-310912

14. Cui C, Zhai D, Cai L, Duan Q, Xie L, Yu J. Long noncoding RNA HEIH promotes colorectal cancer tumorigenesis via counteracting miR-939Mediated transcriptional repression of Bcl-xL. Cancer Res Treat. 2018;50(3):992-1008. doi:10.4143/crt.2017.226

15. Yan Y, Wang Z, Qin B. A novel long noncoding RNA, LINC00483 promotes proliferation and metastasis via modulating of FMNL2 in CRC. Biochem Biophys Res Commun. 2019;509(2):441-447. doi:10.1016/j.bbrc.2018.12.090

16. Zhong X, Lu M, Wan J, Zhou T, Qin B. Long noncoding RNA kcna3 inhibits the progression of colorectal carcinoma through down-regulating YAP1 expression. Biomed Pharmacother. 2018;107:382-389. doi:10.1016/j.biopha.2018.07.118

17. Xiao W, Wang C, Chen K, et al. MiR-765 functions as a tumour suppressor and eliminates lipids in clear cell renal cell carcinoma by downregulating PLP2. EBioMedicine. 2020;51:102622. doi:10.1016/ j.ebiom.2019.102622

18. Xie BH, He X, Hua RX, et al. Mir- 765 promotes cell proliferation by downregulating INPP4B expression in human hepatocellular carcinoma. Cancer Biomark. 2016;16(3):405-413. doi:10.3233/ CBM-160579

19. Ding Z, Jian S, Peng X, et al. Loss of MiR-664 expression enhances cutaneous malignant melanoma proliferation by upregulating PLP2. Medicine. 2015;94(33):e1327. doi:10.1097/MD.0000000000001327

20. Li X, Luo Y, Liu L, et al. The long noncoding RNA ZFAS1 promotes the progression of glioma by regulating the miR-150-5p/PLP2 axis. J Cell Physiol. 2020;235(3):2937-2946. doi:10.1002/jcp.29199

21. Zou Y, Chen Y, Yao S, et al. MiR-422a weakened breast cancer stem cells properties by targeting PLP2. Cancer Biol Ther. 2018;19 (5):436-444. doi:10.1080/15384047.2018.1433497

\section{Publish your work in this journal}

Cancer Management and Research is an international, peer-reviewed open access journal focusing on cancer research and the optimal use of preventative and integrated treatment interventions to achieve improved outcomes, enhanced survival and quality of life for the cancer patient.
The manuscript management system is completely online and includes a very quick and fair peer-review system, which is all easy to use. Visit http://www.dovepress.com/testimonials.php to read real quotes from published authors. 